\title{
Time Constraints and Resource Sharing in Adults' Working Memory Spans
}

\author{
Pierre Barrouillet and Sophie Bernardin \\ Université de Bourgogne
}

\author{
Valérie Camos \\ Université René Descartes Paris V
}

\begin{abstract}
This article presents a new model that accounts for working memory spans in adults, the time-based resource-sharing model. The model assumes that both components (i.e., processing and maintenance) of the main working memory tasks require attention and that memory traces decay as soon as attention is switched away. Because memory retrievals are constrained by a central bottleneck and thus totally capture attention, it was predicted that the maintenance of the items to be recalled depends on both the number of memory retrievals required by the intervening treatment and the time allowed to perform them. This number of retrievals:time ratio determines the cognitive load of the processing component. The authors show in 7 experiments that working memory spans vary as a function of this cognitive load.
\end{abstract}

Ever since the seminal studies by Baddeley and Hitch (1974), working memory has generally been viewed as a system devoted to the coordination of processing and storage. The authors observed that when adults were asked to perform a reasoning task, their performances were impaired by a concurrent memory load and that this impairment was all the more pronounced the more difficult the reasoning task was. Thus, Baddeley and Hitch (1974) suggested that both processing and storage involve a common cognitive system that they thought of as a central executive. The limited capacity of this central executive is thought to be responsible for the phenomenon known as the processing-storage tradeoff. Under heavy memory load, resources that are devoted to storage are no longer available for processing and performance deteriorates.

This resource-sharing conception was directly responsible for the creation of complex span tasks that aimed to evaluate working memory capacities. Contrary to simple span tasks that require only storage (e.g., the digit or the letter spans), working memory span tasks involve both processing and storage (e.g., the reading or the counting spans; Case, Kurland, \& Goldberg, 1982; Daneman \& Carpenter, 1980). In line with the hypothesis that working memory spans measure some resource-sharing capacity within a central executive involved in complex cognitive activities, working memory spans have proven to be highly predictive of performance in

Pierre Barrouillet and Sophie Bernardin, Laboratoire d'Etude de l'Apprentissage et du Développement, Université de Bourgogne, Dijon, France; Valérie Camos, Laboratoire Cognition et Développement, Université René Descartes Paris V, Paris, France.

Parts of this work were presented at the First European Working Memory Symposium, Ghent, Belgium, September 2002, and at the 13th European Society of Cognitive Psychology Conference, Granada, Spain, September 2003. We thank Aude Mariller and Magalie Girardon for their help in running Experiment 1.

Correspondence concerning this article should be addressed to Pierre Barrouillet, Laboratoire d'Etude de l'Apprentissage et du DéveloppementCentre National de la Recherche Scientifique 5022, Université de Bourgogne, Pôle AAFE, Esplanade Erasme, BP 26513, 21065 Dijon, France. E-mail: barouil@u-bourgogne.fr reading comprehension (Daneman \& Carpenter, 1980, 1983), the processing of ambiguous syntactic constructions (Miyake, Just, \& Carpenter, 1994), reasoning (Barrouillet, 1996; Kyllonen \& Christal, 1990), and complex learning (Shute, 1991). Thus, several models assume that the limitations of working memory stem, at least in part, from the limited amount of cognitive resources that can be seen either as sources of activation or as attentional energy (Lovett, Reder, \& Lebière, 1999), capacities for controlled attention (Engle, Kane, \& Tuholski, 1999), or capacities for supervising and coordinating multiple-system functioning (Baddeley, 1990).

However, the resource-sharing hypothesis has recently been called into question by Towse and Hitch, who have argued that, in children (Towse \& Hitch, 1995; Towse, Hitch, \& Hutton, 1998) as well as in adults (Towse, Hitch, \& Hutton, 2000), working memory spans do not primarily depend on the difficulty but on the duration of the processing component of the working memory span tasks. In the same way, Kieras, Meyer, Mueller, and Seymour (1999) have suggested in their EPIC model that working memory limitations do not result from a limited supply of general cognitive resources but from the decay of symbolic codes. Thus, Towse and Houston-Price (2001) concluded that "it is increasingly apparent that both theoretical and computational accounts make the idea of limited resource-sharing capacity superfluous" (p. 246).

Should we really abandon the notion of resource-sharing capacity and, more generally, any notion of resource in accounting for working memory spans? The aim of this article was to answer this question. First, we present Towse and Hitch's (1995) taskswitching model as well as the results of our previous study (Barrouillet \& Camos, 2001) that contradicted their hypotheses. Following our 2001 suggestions, we then propose an alternative model for working memory span tasks that we call the "time-based resource-sharing" model. This model fully integrates time constraints and resource-sharing processes into working memory span tasks, but it goes beyond a purely metaphorical conception of resource sharing by providing a metric of the cognitive load (CL) a given task involves. This model was tested in two series of experiments in which we used new working memory span tasks that enabled us to carefully control for both the nature and the duration of the tasks. In a first series, we demonstrated that adults' 
spans depend mainly on the cognitive cost imposed by the processing component of the working memory tasks, whereas the total duration has only a moderate, if any, effect. In a second series of experiments, we tested more specific aspects of our model, and we provide evidence that working memory span is a function of both the number of memory retrievals the processing component requires and the time allowed to perform them.

\section{Task Switching, Memory Decay, and CL}

Case et al. (1982) suggested that the processing-storage tradeoff that results from resource-sharing is responsible for the developmental increase they observed in counting span. ${ }^{1}$ Due to automatization and practice, the counting activity becomes less and less resource demanding with age, thus leaving more resources available for storage. However, Towse and Hitch (1995) put forward an alternative "memory decay" hypothesis: The strength of the memory traces in the short-term storage space weakens as the temporal interval between storage and recall increases. Thus, the higher counting span of older children might be due to the fact that they count the arrays of dots more quickly and that this speed reduces the lapse of time during which the information has to be retained. Towse and Hitch tested this hypothesis by manipulating the difficulty and the duration of the counting component of a counting span task in children. They observed that a more difficult counting activity (reduced discriminability between the targets to be counted and the distracters) did not result in lower spans, whereas longer counting durations did. The authors concluded that counting spans do not depend on the amount of workspace required by the counting but on the duration of the counting during which the totals may be forgotten. Thus, they suggested that children may switch between the counting process during the display presentation and storing results at the end of each counting.

Towse et al. (1998) tested this task-switching model in a series of experiments in which children were presented with sequences of cards to count. On these cards, the array numerosity of the last card was either small (and that of the first card large) or large (and that of the first card small), the large-final condition involving a longer retention period than that of the small-final condition. As they predicted, recall performances were poorer in the former than in the latter condition, providing evidence that spans rely on retention period and not on cognitive demand. These results have been extended to reading and operation span tasks, in children as well as in adults (Hitch, Towse, \& Hutton, 2001; Towse et al., 2000).

However, in Barrouillet and Camos (2001), we argued that when manipulating the duration of counting or operation solving, Towse and Hitch also modified the cognitive cost of these activities. Thus, they suggested comparing children's performances in tasks in which the processing component retained the same duration but varied in cognitive cost. For example, they compared a counting span and an operation span, in which children were asked to remember letters presented at the end of each array to be counted or operation to be solved, with performance in a similar task, in which children were asked to say "ba-ba" repeatedly instead of counting dots or solving operations but over the same period of time. This concurrent articulation was intended to prevent any rehearsal strategy. As predicted by the memory decay hypothesis, they did not find any difference between counting and baba spans, but the operation span was systematically lower than the baba span. From this last result, Barrouillet and Camos concluded that Towse et al.'s (1998) task-switching model was an oversimplification. Indeed, according to this latter model, there should be no difference between operation span and baba span because the interletter durations were held constant between the two tasks. Thus, Barrouillet and Camos suggested integrating both time and resource constraints into a model in which participants switch their attention from processing to storage during processing. The aim of this article was to elaborate and test such a model.

\section{A Time-Based Resource-Sharing Model}

The time-based resource-sharing model is based on four main proposals. First, we assume that both components (i.e., processing and maintenance) of most working memory span tasks require attention (e.g., reading span, operation span, counting span, alphabet recoding, $\mathrm{ABCD}$ tasks). Because attention constitutes a limited pool of resources, it must be shared between processing and maintenance. Second, as soon as attention is switched away from the memory traces of the items to be recalled, their activation suffers from a time-related decay. Refreshing these decaying memory traces requires their retrieval from memory by attentional focusing. Third, although there may be multiple ways in which processing tasks switch attention away from maintenance, the tasks that occupy the retrieval process needed to refresh memory traces should have an especially detrimental effect on maintenance because there is a central bottleneck that constrains retrieval activities. Fourth, when the processing component involves retrievals, sharing attention is time-based because the central bottleneck allows only one memory retrieval at a time. Thus, attention sharing is achieved through a rapid and frequent switching between processing and maintenance that occurs during the completion of the task.

As far as the first point is concerned, we assume that processing and maintenance rely on the same, limited attention resource. Most of the main working memory span tasks involve complex activities as processing components (e.g., reading sentences, operation solving, counting, alphabetic arithmetic, reasoning). These processing components require planning multistep strategies, setting goals and subgoals, and maintaining intermediary results, all activities that obviously require controlled attention (Engle et al., 1999). However, reading sentences, solving arithmetic problems, or even counting dots also involve series of retrievals from long-term memory. Within the ACT-R model (Anderson, 1993; Anderson \& Lebiere, 1998), the retrieval of declarative knowledge is achieved through an activation process that results from attentional focusing on retrieval cues. It should be noted that within this theoretical framework, even the simplest goal-directed retrieval (e.g., to retrieve the answer to a small and frequent addition) requires attentional resources (Cowan, 1999; Lovett et al., 1999). Thus, even the elementary steps involved in the tasks most frequently used as processing components in working memory span tasks require attention. In line with many models of working memory (Baddeley, 1996; Cowan, 1995, 1999; Engle et al., 1999), the ACT-R

\footnotetext{
${ }^{1}$ In the counting span task, children are asked to count out loud dots on cards and then recall the number of dots present on each card. The counting span is the maximum number of cards the participant is able to remember.
} 
model assumes that this attentional resource for activation constitutes a limited pool whose capacity differs between individuals (Lovett et al., 1999). Moreover, many models of working memory suggest that short-term memory is that part of long-term memory that is activated above threshold (Anderson, 1993; Cantor \& Engle, 1993; Cowan, 1995; Engle \& Oransky, 1999; Lovett et al., 1999) and that working memory contains items that are outside of the focus of attention but sufficiently activated to be readily accessible (Conway \& Engle, 1994; Cowan, 1995). The activation of those items from long-term memory that constitute short-term memory is achieved by attentional focusing (Cowan, 1995, 1999). Thus, the maintenance in short-term memory of the items to be recalled also requires attention. As a consequence, both components of working memory span tasks depend on the same resource (i.e., attention) needed to activate knowledge and to maintain active goals, intermediary results, and the memory traces of the items to-be-remembered. As a consequence, the completion of the working memory span tasks requires some sharing of this unique resource between processing and storage.

Second, according to Cowan (1995), the items present in the focus of attention receive activation, but this activation decays as soon as they leave the focus. Thus, the memory traces of the items to be recalled suffer from a time-related decay as soon as attention is switched away from maintenance (Baddeley \& Logie, 1999; Cowan, 1999). Refreshing these memory traces during the completion of the working memory span task would involve their retrieval by attentional focusing before their complete disappearance. Because working memory span tasks impose resource sharing between processing and storage, this decay would be a function of the time during which the concurrent processing totally captures attention and thus impedes refreshing.

This is true of any working memory span task in which the processing component does not require memory retrievals but involves attentional demand, because refreshing the decaying memory traces of the stored items requires attention to effect their retrieval. Thus, any processing component that captures some part of the available attention capacity should impede the retrievals on which the maintenance of the memory items relies. For example, Rohrer and Pashler (2003) demonstrated that a secondary task that does not involve verbal material and does not rely itself on retrievals but that requires attention (a serial choice-reaction time task) reduces speed and accuracy of recall in a concurrent freerecall task. The authors conclude that memory retrieval is subject to severe interference from unrelated central processing. However, and this is our third point, this effect would be particularly pronounced when the processing component involves memory retrievals, which is the case in the main verbal working memory span tasks.

As we have emphasized previously, attention is needed to achieve the successive retrievals involved in both processing and storage components. However, following Pashler (1998), we suppose that there is a central bottleneck that constrains retrieval activities. In effect, as Rohrer, Pashler, and Etchegaray (1998) demonstrated, two memory retrievals cannot be performed simultaneously. To account for this fact, the authors suggested the metaphor of a spotlight of retrieval that would be directed at only one category at a time in such a way that multiple categories can simultaneously remain activated for a short while, but only one category can be lit. Similarly, Garavan (1998) concluded from a study on attention-switching processes that individuals can attend to just one "object" in working memory at any one time.

Many experimental results suggest that, among attentiondemanding activities, those that have the most detrimental effect on retrievals are precisely other concurrent memory retrievals. For example, De Rammelaere, Stuyven, and Vandierendonck (1999) compared the effect of a random time interval generation (RIG), a random letter generation (RLG), and a concurrent articulation on the verification of simple arithmetic problems known to be solved by direct retrieval of the answer from memory. They observed that the RIG, which is attention demanding, elicited an increase of about $200 \mathrm{~ms}$ in the verification times (see also, De Rammelaere, Stuyven, \& Vandierendonck, 2001). However, the RLG led to a dramatic increase of $1,300 \mathrm{~ms}$ in verification times whereas a simple concurrent articulation had no effect. This result suggests that the successive memory retrievals involved in the RLG not only capture attentional resources as RIG did but also block the spotlight of retrieval needed to verify the arithmetic problems. Thus, in a working memory span task, processing components that involve retrievals should be the most disruptive for the refreshing of the items to-be-remembered.

According to our previous analyses, both components of most working memory span tasks require series of retrievals that compete for a unique spotlight of retrieval. We assume that, in this case, the sharing of attention is achieved through a process of rapid switching of the focus from one component of the span task to another. However, there is no need to suppose that, during the memory span task, this switching only occurs when the participants are presented with a new item to memorize or with a new problem to solve, as Towse et al. (1998) suggested. As demonstrated by Cowan in the field of recall activities (Cowan, 1992; Cowan et al., 1994), individuals can engage in a covert retrieval process during short pauses to reactivate decaying memory traces. In the same way, it may well be supposed that, while engaged in the counting, operation solving, or reading component of working memory span tasks, participants may keep short pauses free for the brief reactivation of the memory items by means of a simple mental search without engaging in time-consuming activities such as rehearsal.

\section{A New Conception and a Metric of Cognitive Cost}

It should be noted that our model delineates a conception of cognitive cost that departs from more traditional conceptions that conflate cognitive cost with complexity. Usually, the concurrent tasks used in working memory span tasks involve activities that are considered costly because, within the resource-sharing framework, a precise measure of cognitive resources is thought to be achieved when the concurrent task captures a substantial proportion of cognitive capacity. It is generally assumed that this condition is fulfilled by activities that involve multistep processes, the integration of several sources of information, or the control of processing and intermediate results - in other words by complex activities such as counting, operation solving, alphabetic arithmetic, text comprehension, or reasoning. It has been observed that this kind of concurrent task has a highly detrimental effect on recall. However, the fact that complex concurrent activities produce the intended effect on recall does not mean that this effect is due to complexity. 
On the contrary, our model predicts that complex concurrent activity is not needed.

Our conception of cognitive cost involves time constraints as a main factor. For a given period of time, the cognitive cost that a given task involves is a function of the time during which it captures attention in such a way that the refreshing of decaying memory traces is impeded. The longer this time, the fewer and shorter the periods during which pauses can be kept free to divert attention from processing to the retrieval and the refreshing of decaying memory traces of the items to be recalled. Note that this time is probably highly difficult to determine for any given task, however elementary it may be, and that it does not merely correspond to its raw duration. As pointed out by Rohrer and Pashler (2003), the centrally demanding stages of an activity are virtually certain to occupy no more than a sizable fraction of the time consumed by this activity. For example, in the case of memory retrievals, this time would correspond to that during which the central bottleneck is fully occupied. Thus, for a processing component that mainly involves retrievals from memory, this time would be a function of both the number and difficulty of the retrievals to be performed in a given period. It is assumed that some memory retrievals are more difficult than others because they occupy the spotlight of retrieval for a longer period of time, something that is reflected in measures like reaction or response times. It is, for example, well known that fact numbers are easier and faster to retrieve when they involve small rather than large operands (Aschcraft \& Battaglia, 1978; Siegler \& Shrager, 1984) or, in the same way, that words are better retrieved as their frequency increases (Monsell, 1991). These facts suggest that retrievals differ in the duration of their centrally demanding stages.

Thus, according to our previous analyses, any task that requires an uninterrupted series of retrievals from long-term memory should result in a heavy CL and should have a highly detrimental effect on recall when inserted into a working memory span task. For this kind of task, CL, that is the time during which attention is captured, would be a function of the number of retrievals, their nature, and the total time allowed to perform them. When different retrievals are performed at a constant pace, the CL would correspond to the following:

$$
\mathrm{CL}=\sum a_{i} n_{i} / T,
$$

where $n_{i}$ corresponds to the number of retrievals of the type $i, a_{i}$ to a parameter that represents the difficulty of these retrievals $i$, that is to say the time during which these retrievals totally capture attention, and $T$ to the total duration of the activity. In a simplified situation in which all the retrievals are identical in nature (i.e., present similar parameter $a$ ), the cognitive load would correspond to the following:

$$
\mathrm{CL}=a N / T \text {. }
$$

In this latter case, cognitive load can be assimilated to the number of retrievals:time ratio. Suppose that participants are asked to maintain letters in memory while reading aloud digits that are sequentially presented on the screen. Provided that the digits are presented at a comfortable pace, this activity could allow participants to free up interdigit pauses during which the central bottleneck for retrieval is available for the retrieval and updating of the decaying memory traces (see Figure 1a). This would result in high

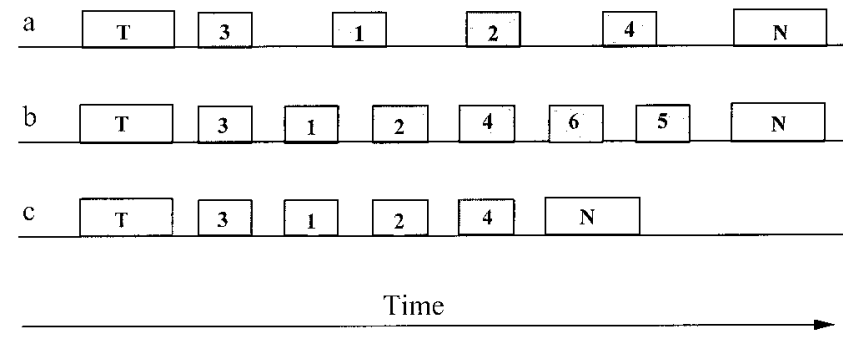

Figure 1. Schematic representation of the interletter interval in a working memory span task in which participants have to maintain letters in memory while reading digits aloud (a) and modifications resulting either from the increase in the number of intervening digits (b) or from the reduction of the interletter interval (c).

spans. However, any increase in the number of digits to be read results in an increase in the number of retrievals:time ratio and a concomitant shortening of the interdigit pauses (see Figure 1b). This would result in poorer recalls and lower spans. The same phenomenon would be observed when, instead of increasing the number of digits presented, the total time allowed to perform the task is reduced (see Figure 1c). In both situations, the task in question remains quite simple (i.e., reading digits), but our model predicts that its cognitive load will become considerable as this depends on the pace of retrieval.

\section{A Comparison Between the Time-Based Resource-Sharing and the Task-Switching Models}

Although both the time-based resource-sharing and the taskswitching models rely on a switching process, they lead to opposite predictions because they differ on two main points. First, our model postulates a resource-sharing process, and, second, this process is time-based and relies on a rapid switching that occurs during processing.

As far as the first point is concerned, our model predicts that when the total duration of the task is held constant, working memory span should vary as a function of the cognitive load the processing component involves. This prediction, which had already been tested in children by Barrouillet and Camos (2001), was tested in adults in three experiments. The first experiment replicated the comparison between the operation span and $b a b a$ span that Barrouillet and Camos had previously performed in children. In Experiment 2, we designed two new computer-paced tasks that permit careful control of duration. In the first, participants were asked to solve simple additions or subtractions while maintaining series of letters (the continuous operation span task). In the second, the participants were simply asked to read aloud the same operations, the answers to which were displayed on screen (the reading operation span task). These two spans were compared with a baba span. In Experiment 3, the same tasks were used but two different durations (short and long) of the processing components were used in a factorial design.

In these first three experiments, our theory predicts that, when retention periods are held constant, working memory span should vary as a function of the cognitive cost of the processing component of the tasks. Thus, operation and continuous operation spans should be lower than baba span, whereas the reading operation 
span task should result in an intermediate level of performance. The continuous operation span involves a series of retrievals of the answer to each operation. We assume that these retrievals are more demanding than those involved in the reading operation span task because they are more susceptible to interference from other answers to related operations that share the same operands (Zbrodoff \& Logan, 1996). By contrast, the reading operation span task involves the retrievals of phonological representations that are directly cued by the digital forms presented on screen. These retrievals should thus leave more room for refreshing processes. As far as the baba span is concerned, this task simply involves the retrieval of a unique phonological representation from the recursive presentation of an unchanged stimulus (the syllable $b a$ presented on screen). This representation should thus remain highly activated and very accessible. As a consequence, it is assumed that articulatory suppression should involve shorter centrally demanding stages than those of solving or reading operations and so a lower cognitive cost and higher spans. By contrast, Towse et al.'s (1998) task-switching model predicts no difference among the three tasks because their total duration is the same, whereas the cognitive cost of the processing activity does not matter. This theory only predicts an effect of the duration of the tasks in Experiment 3 with lower working memory spans associated with longer durations.

The second main difference between our theory and the taskswitching model concerns the effect of time. According to the task-switching model, retention periods have a straightforward effect on spans: Longer periods result in lower spans. By contrast, in our model, cognitive load mediates the effect of time. When the number of memory retrievals that the processing component requires is held constant, while the time allowed to perform them is reduced (see Figure 1c), our model predicts lower spans. This is due to the fact that, provided that the time needed to perform the retrievals is, broadly speaking, incompressible, shorter duration results in a reduction of the possible pauses during which decaying memory traces can be retrieved and restored. The same phenomenon should result from increasing the number of retrievals to be performed in an unchanged total period of time.

A second series of three experiments tested this general hypothesis. In Experiment 4, we tested the effect of the number of retrievals while the total duration was held constant using a reading-digit span task in which the processing component consisted of a reading-digit task while participants were asked to maintain series of letters in memory. Experiments 5 and 6 tested the more provocative prediction that shorter periods of retention would result in lower spans. In Experiment 5, we used a readingdigit span task in which the number of digits to be read between each letter to be remembered was held constant while the interletter interval was reduced. Experiment 6 followed the same logic but used a continuous operation span task. In these last two experiments, our theory predicts that a shorter total duration of the activity will result in a higher cognitive load and thus in lower working memory spans, whereas Towse et al.'s (1998) taskswitching model predicts the opposite effect. This model only considers the raw duration of the interpolated task because it disallows a switching during the completion of the processing component. For example, in the counting span task, it is assumed that "there is no active maintenance of stored totals that competes with the execution of counting operations" (Hitch et al., 2001, p.
185). According to the authors, participants do not attempt to maintain memory items during processing, but switch between phases of activity devoted to either processing or retention, reflecting the way the working memory span tasks are structured (Towse, Hitch, \& Hutton, 2002).

Finally, a last experiment integrated both the resource-sharing and time-based aspects of our theory. The resource-sharing theory predicts that when resources are reallocated from storage to processing, recall performance deteriorates. According to Equation 2 for cognitive load, the processing demand is a direct function of the number of retrievals:time ratio. Thus, progressively increasing this ratio should result in a smooth and linear decrease in span, this decrease being all the more pronounced as the difficulty parameter $a$ is high.

\section{Experiment 1}

The aim of this experiment was to replicate and extend previous results observed in children by Barrouillet and Camos (2001). To test their cognitive load hypothesis against Towse and Hitch's (1995) memory decay hypothesis, Barrouillet and Camos compared Turner and Engle's (1989) operation span with the baba span while holding the total duration of both tasks constant. As predicted, the authors observed that the processing component involving a higher cognitive load (i.e., operation solving) led to lower recall performance and that the operation span was lower than the baba span. However, this difference was highly significant in 9-year-old children but decreased and failed to reach significance in 11-year-old children. It could thus be argued that the cognitive load hypothesis holds only in children who lack the appropriate skills to perform the tasks efficiently. As soon as these skills are acquired, the putative cognitive load would no longer matter and the duration of the task would be the sole relevant factor for working memory spans, as predicted by the memory decay hypothesis (Towse \& Hitch, 1995).

The present experiment was designed to test this possibility. On the one hand, if Towse et al.'s (1998) task-switching model is correct, and if the difference between the operation span and the baba span is a short-lived phenomenon restricted to a circumscribed developmental period, then there should no longer be a difference in adults when the duration of the retention periods is held constant between the two tasks. On the other hand, if our time-based resource-sharing model is correct, there should still be a difference between the operation span and the baba span in adults because solving operations is more attention demanding than repeatedly saying "ba-ba." The rationale of the present experiment was the same as that used by Barrouillet and Camos (2001). The first group performed a self-paced operation span task (Turner \& Engle, 1989) in which participants were presented with letters to be remembered, each followed by an operation to be solved in series of increasing length. The mean time needed to solve each of these operations was calculated. A second group of participants was then presented with the same series of letters and asked to keep saying the syllable "ba" over the same period that it took for the corresponding operation to be solved in the first group. We predicted that, although the duration of the retention period was held constant between the two tasks, the mean operation span in the first group would be lower than the mean baba span in the second group. 


\section{Method}

Participants. A total of 49 undergraduate psychology students at the Université de Bourgogne (Dijon, France) received a partial course credit for participating and were randomly assigned to the $b a b a$ span and the operation span groups.

Material and procedure. The material and procedure were the same as in Barrouillet and Camos (2001). Both tasks were presented on screen. As far as the operation span task was concerned, participants were presented with series of increasing length (from one to six operations) in which each operation was preceded by a consonant to be remembered. There were three series of each length. The operations were 54 three-operand additions (e.g., $4+7+8$ ) and 9 two-operand additions (e.g., $9+8$ ). Half of these problems had a correct answer (e.g., $4+7+8=19$ ), and the other half had an incorrect answer (correct answer \pm 1 ). These problems had been selected from a pool of about 90 additive problems, had elicited a correct response of over $80 \%$, and did not take longer than $14 \mathrm{~s}$ to be solved by third graders. The 63 problems were randomly assigned to the 18 series.

All the series had the same structure. First, the participants were asked to focus for $500 \mathrm{~ms}$ on a signal (an asterisk) centered on the screen that was replaced, for a period of $1 \mathrm{~s}$, by a letter that they were asked to remember. When the letter disappeared, a problem was displayed on screen. The participants had to evaluate the answer and give their response (true or false) by pressing one of two keys labeled on the computer keyboard. Reaction times and type of response were recorded. As soon as the participant pressed a key, the problem was replaced by the signal and a new trial began. At the end of the series, the word Rappel (recall) was displayed on screen and the participants were asked to recall the letters in the correct order. The experimental trials were preceded by three 1-letter and three 2-letter training series.

As far as the baba span task was concerned, the sequence of events was the same except that the problems to be verified were replaced by empty screens. The duration of presentation of each empty screen was the mean time recorded in the operation span group for verification of the corresponding problem. During the presentation, the participants were asked to say the syllable "ba" on each beat of a metronome (one beat per $600 \mathrm{~ms}$ ). Before performing the task, the participants were familiarized with the rhythm of the metronome and performed three 1-letter and three 2-letter series.

For both tasks, and following Daneman and Carpenter's (1980) procedure, participants were presented with increasingly long series of letters until they failed to recall the letters of all three series at a particular level. Testing was terminated at this point. Each correctly recalled series counted as one third; the total number of thirds was added up to provide a span score (Kemps, De Rammelaere, \& Desmet, 2000; Smith \& Scholey, 1992). For example, the correct recall of all the series of one, two, and three letters, of two series of four letters and one series of five letters resulted in a span of $(3+3+3+2+1) \times 1 / 3=4$.

\section{Results}

To ensure that the participants in the operation span group paid sufficient attention to the task and did not neglect the operationsolving activity, those participants who exhibited a rate of correct responses lower than $75 \%$ were discarded from the analyses. This procedure removed only 1 participant (rate of correct responses $73 \%$, operation span: 3.33 ). The remaining 24 participants had a mean rate of correct responses of $92 \%(S D=6 \%)$. As far as the baba span was concerned, all 24 participants succeeded in conforming to the rhythm of pronunciation imposed by the metronome. The mean solving times of the 63 problems presented in the operation span group, and thus the mean presentation times of empty screens in the baba span group, ranged from 2,577 ms
$(S D=735 \mathrm{~ms})$ for the shortest to $7,438 \mathrm{~ms}(S D=3,216 \mathrm{~ms})$ for the longest.

As predicted by the resource-sharing hypothesis, the mean $b a b a$ span $(4.58, S D=0.76)$ was significantly higher than the mean operation $\operatorname{span}(3.49, S D=1.21), t(46)=3.76, p<.01, \eta^{2}=$ .235 . This result contradicts the memory decay hypothesis and the task-switching model, which predicted no difference between the two spans, because in both tasks the retention period of the letters before recall was held constant. Moreover, the significant difference we observed indicates that the effect of cognitive load on span is not restricted to the developmental period but extends to adulthood. Of course, this first result is not sufficient to rule out the task-switching model. It could be argued that the duration of the retention period was not carefully balanced between the two tasks because this period remained absolutely unchanged between participants in the baba span group, whereas it varied from one participant to another in the operation span group. Actually, what is required is an operation span task that is not self-paced but permits careful control of duration. Such a task is presented in the following experiment.

\section{Experiment 2}

As pointed out previously, the self-paced character of the standard working memory span tasks is a major shortcoming when comparisons of duration are required. However, it is not possible to constrain the time allowed to solve the complex operations involved in Turner and Engle's (1989) task. On the one hand, solution times vary greatly from one participant to another (standard deviations of more than $3 \mathrm{~s}$ for mean solving times of about $7 \mathrm{~s}$ in Experiment 1), and many participants would not have enough time to perform the task if major time constraints were imposed. On the other hand, allowing excessively long periods in which to solve the operations would leave many of the fastest participants free to covertly rehearse the items to be remembered, thus undermining the rationale of the tasks. Thus, we designed new working memory tasks in which the processing component involves simpler activities that present lower interindividual variability and whose duration can be fixed by the experimenter. Moreover, it could be argued that the difference between operation spans and baba spans is not really indicative of the processes involved in working memory span tasks because the tasks we compared are so different (operation solving vs. a simple concurrent articulation) that the locus of the observed effects remains unclear. What is required are tasks that involve more comparable activities.

In consequence, we designed a first new working memory span task in which participants, while maintaining letters in memory, were asked to solve successive simple operations instead of complex equations. For example, instead of being presented with a complex problem like " $4+7+8=19$ ?" after each letter to be remembered, they were presented with small operations whose operands appeared successively on screen for short periods of time (e.g., $4 /+1 /-2 /+1)$. They were asked to solve these operations aloud, thus saying " 4 ," "plus 1, 5," "minus 2, 3," "plus 1, 4." We called this new computer-driven task the continuous operation span task. We compared this continuous operation span with two other spans from working memory tasks in which the duration of the treatments, and thus the retention periods, were 
exactly equivalent. The first was the previously presented $b a b a$ span task in which the number of $b a$ syllables to be spoken was determined by the number of syllables involved in solving the corresponding operations in the continuous operation span task. The second task was designed to achieve a perfect matching of both the retention periods of the letters to be remembered and the level of concurrent articulation involved. Thus, the participants were presented with the same operands as in the continuous operation span task but also with their successive answers that they simply had to read aloud. For example, instead of solving $4 /+1$ $/-2 /+1$, they read aloud " $4 /+1 / 5 /-2 / 3 /+1 / 4$," thus pronouncing exactly the same words within the same period of time. We called this task the reading operation span task.

As previously argued, it is more attention demanding to perform than to read successive arithmetic operations because the former activity involves more demanding retrievals that suffer from more interference than the latter and thus occupies the central bottleneck for longer periods of time. Thus, despite the fact that both tasks had exactly the same duration and required the pronunciation of exactly the same words, we predicted that the continuous operation span would be lower than the reading operation span. This latter would, in turn, be lower than the baba span, which requires only the successive reactivation of a unique phonological trace of the syllable $b a$. By contrast, the task-switching model predicts no difference between the three spans because the duration of the retention periods was carefully balanced within and among the three tasks.

\section{Method}

Participants. Seventy-two undergraduate psychology students at the Université de Bourgogne received a partial course credit for participating and were randomly assigned to the continuous operation span, the reading operation span, or the baba span group. None of them had participated in the previous study.

Material and procedure. As far as the continuous operation span task was concerned, the participants were presented with series of ascending length (from 1 to 7 continuous operations) in which each operation was preceded by a consonant to be remembered. There were three series of each length. The 84 continuous operations had the same structure. They involved first a one-digit operand, which we called the root, followed by a string of two to four sign-operand pairs in which the sign was either plus or minus and the operand either one or two. Each number from one to nine was used as a root in a roughly equivalent number of continuous operations (i.e., 9 or 10 times) and associated with random strings of sign-operand pairs. However, those strings that led to intermediate or final answers lower than zero or higher than nine were discarded (e.g., $7 /+2 /+1 /-1 /$ +2 , or $3 /-2 /-2$ ). Moreover, for each length of the letter series, there was the same number of continuous operations with two, three, and four sign-operands pairs, and each of the four possible pairs (i.e., $+1,+2,-$ 1 , and -2) appeared with the same frequency.

All the series had the same structure. First, the participants were asked to focus for $750 \mathrm{~ms}$ on a signal (an asterisk) centered on the screen, which was replaced, after a delay of $500 \mathrm{~ms}$ and for a period of $1,500 \mathrm{~ms}$, by a letter to be remembered. This letter was immediately followed by a number (a root) for a period of $1 \mathrm{~s}$, and then by a string of sign-operand pairs, each of them remaining on screen for $2 \mathrm{~s}$. Thus, the interletter intervals were of $5 \mathrm{~s}, 7 \mathrm{~s}$, or $9 \mathrm{~s}$ for continuous operations containing strings of two, three, and four sign-operand pairs respectively. Preliminary tests indicated that, while requiring sustained attention, this rhythm of presentation was acceptable for adults. To avoid any confusion between two consecutive sign-operand pairs, we centered the first pair on screen, whereas the following pairs progressively shifted to the right. The last pair was displayed in red, thus signaling the end of the operations and the next appearance of a new letter to be remembered. The participants were asked to read the letters and to perform the operations aloud. At the end of the series, the word Rappel [recall] was displayed on screen, and the participants were asked to recall the letters in the correct order.

The material and procedure were exactly the same for the reading operation span task except that the answer to each operation was displayed on screen. Thus, instead of presenting sign-operand pairs for $2 \mathrm{~s}$, we presented each pair for $1 \mathrm{~s}$ followed by the corresponding answer for another second. For example, instead of " $4 /+1 /+2$," the successive screens presented were " $4 /+1 / 5 /+2 / 7$." Thus, the total duration of the intervening events that occurred between the presentations of two letters was precisely the same in the continuous operation and the reading operation span tasks. The answers occupied the same position on screen and were of the same color (blue, but red for the final values) as the associated sign-operand pair. The participants were asked to read the successive screens aloud.

The baba span used the same series of letters, but the letters "BA" (syllable $b a$ ) were displayed on screen instead of numbers and signoperand pairs. Each presentation of the syllable was preceded by a delay to avoid any confusion between the successive pronunciations. Because continuous operations with two, three, and four sign-operands lasted $5 \mathrm{~s}, 7 \mathrm{~s}$, and $9 \mathrm{~s}$ and required the participants to say 7, 10, and 13 syllables, respectively, they were replaced by an equal number of presentations of the syllable $b a$ that lasted $714 \mathrm{~ms}, 700 \mathrm{~ms}$, and $692 \mathrm{~ms}$, respectively. During each of these presentations, the screen remained empty half of the time, then the syllable appeared. The syllables occupied the same position on screen and were of the same color as the associated sign-operand pair. For example, the last three $b a$ s were red and appeared in the rightmost position. The participants were asked to say "ba" each time the syllable appeared on screen.

For each task, three 1-letter and three 2-letter training series preceded the experimental series. As in Experiment 1, the tasks were interrupted as soon as participants failed to recall the letters on each set of a given length series correctly. The spans were calculated as in Experiment 1.

\section{Results}

Despite observations from preliminary tests indicating that the rhythm of presentation of the continuous operations was suitable for adults, some participants failed to comply with this constraint in certain series and interrupted processing (1.8\% of the continuous operations presented). These series were not included in the analyses and were considered as false (5.2\% of the series presented). Indeed, interrupting the task and remaining silent could permit a covert and active rehearsal of the letters to be remembered, thus increasing performance. By contrast, recalls after false but uninterrupted calculations (11\% of the continuous operations) were included. No interruptions or errors occurred in the reading operation span or the baba span tasks.

As we predicted, the mean continuous operation span $(2.60$, $S D=0.63)$ was significantly lower than the mean reading operation $\operatorname{span}(3.15, S D=0.75), t(46)=2.78, p<.01, \eta^{2}=.144$. In line with our predictions, this latter span was, in turn, lower than the baba $\operatorname{span}(4.22, S D=0.75), t(46)=4.93, p<.01, \eta^{2}=.346$. Thus, this experiment leaves us in no doubt about the fact that working memory spans depend to a great extent on the nature of the treatment involved in the processing component of the task. Even when the retention periods of the memory items and the level of concurrent articulation are exactly the same, as was the case in our continuous and reading operation span tasks, treatments in- 
volving longer centrally demanding periods result in lower recall performance. This result strongly supports the resource-sharing hypothesis.

Moreover, our new paradigm allowed us to evaluate the effect of time while holding the difficulty of the task constant. In their earlier studies, Towse and Hitch (1995; Towse et al., 1998, 2000) manipulated the duration of the processing component of the working memory span tasks by presenting longer sentences to be read or longer operations to be solved. However, longer sentences are often more complex and more difficult to understand than short sentences and longer equations often involve larger numbers and more difficult calculations. This is why in Barrouillet and Camos (2001) we claimed that this manipulation of the retention periods also led to changes in the cognitive cost. By contrast, our continuous operation span task, like our reading operation span task, permits a controlled manipulation of duration while keeping the cognitive cost absolutely unchanged. Suppose that we compare continuous operations that involve either two or four sign-operand pairs, each pair being presented for $2 \mathrm{~s}$. The four-pair operations take longer to solve than the two-pair operations ( $9 \mathrm{~s}$ and $5 \mathrm{~s}$, respectively, when the presentation of the root is included), but they do not involve a higher cognitive cost. Indeed, a new signoperand pair that appears on screen does not result in the computation of more complex operations (always plus or minus one or two), the manipulation of larger numbers (always between one and nine), or the retention of more intermediate results in short term memory. It simply involves the lengthening of the activity without modifying its nature or difficulty. Thus, the new working memory span tasks we designed are particularly well suited to testing the effect of the total duration of the task and the predictions of the task-switching model (Towse et al., 1998). This was the object of Experiment 3.

\section{Experiment 3}

The aim of this experiment was to evaluate the effect of the total duration of the processing component on working memory. According to Towse et al.'s (1998) model, the retention period of the memory items has a strong effect on recall because the timerelated decay of memory traces occurs in a simple manner. Beginning as soon as the participant engages in the concurrent task (reading, solving problems, counting arrays), this decay is thought to continue without interruption until the conclusion of processing. It is only when a new item to be remembered is presented or available for storage that participants could refresh the degraded memory traces of previously stored items. Thus, the longer the raw duration of the concurrent task (i.e., the time interval between the presentation of two memory items), the stronger the decay and, at the end of the series, the poorer the recall.

By contrast, our model does not predict that longer tasks will necessarily result in poorer recalls. This hypothesis would only hold in extreme cases in which participants are presented with tasks involving so great a cognitive load that they prevent any possibility of switching attention to the refreshment of memory traces. In other cases, recall performance would depend on the cognitive load as given by Equation 2. As pointed out previously, adding sign-operand pairs to a continuous operation span only lengthens the task without modifying its cognitive cost because the number of retrievals:time ratio remains constant. When cognitive load is kept constant, recall performance should remain unchanged. This is because this performance corresponds to the number of items that rapid switching makes it possible to keep active while completing the concurrent task. Of course, it is conceivable that some level of fatigue could impair maintenance and recall, but the size of this effect should be negligible when compared with the effect of cognitive load. Thus, in Experiment 3, we presented participants with continuous operation span tasks that involved either short (i.e., $5 \mathrm{~s}$, two sign-operand pairs after the root of each continuous operation) or long retention periods (i.e., $9 \mathrm{~s}$, four sign-operand pairs), as well as with the corresponding reading operation span and baba span tasks. The task-switching model predicts a strong effect of duration with higher spans in the short than in the long condition, but with no difference between tasks. Our time-based resource-sharing model makes opposite predictions: a strong effect of task due to differences in cognitive load, as we observed in Experiment 2, and only a moderate, if any, effect of duration.

\section{Method}

Participants. One hundred forty-four undergraduate psychology students at the Université de Bourgogne received a partial course credit for participating. They were randomly assigned to the six experimental groups of 24 participants each defined by the design of 3 Tasks (continuous operation span, reading operation span, and baba span) $\times 2$ Durations (short and long).

Material and procedure. The material and procedure for the three tasks were the same as in Experiment 2. The sole difference was that the number of sign-operand pairs presented after the roots in the continuous operations was fixed at two in the short-duration ( $5 \mathrm{~s})$ and four in the long-duration condition $(9 \mathrm{~s})$. The reading operation span and the baba span tasks in both duration conditions were constructed following the procedure described above. Spans were calculated as in Experiments 1 and 2.

\section{Results}

As in Experiment 2, those recalls that followed interrupted treatments were omitted from the analyses. As far as the continuous operations were concerned, $0.7 \%$ and $3.1 \%$ of the operations presented in the short and long conditions, respectively, were interrupted, thus leading us to omit $1.1 \%$ and $7.9 \%$ of the series, respectively. The reading of the operation was interrupted in only $0.5 \%$ of cases in the long condition ( $1.7 \%$ of the series) and none in the short condition. We performed a 3 (tasks: continuous operation, reading operation, and $b a b a) \times 2$ (durations: short and long) analysis of variance on the spans. As we predicted, and in line with our previous results, there was a strong effect of the tasks, $F(2$, $138)=27.86, p<.01, \eta^{2}=.142$ (see Table 1 ). The mean continuous operation span was lower than the mean reading operation span (2.49 and 3.40, respectively), $F(1,138)=20.18, p<$ $.01, \eta^{2}=.103$, which was, in turn, lower than the baba span (3.99), $F(1,138)=8.50, p<.01, \eta^{2}=.043$. As we anticipated, and contrary to the predictions of the task-switching hypothesis, there was no significant effect of duration, with a short duration even resulting in a directionally lower mean span than the long duration (3.22 and 3.38, respectively), $F<1$. The two factors did not interact, $F<1$. It should be noted that these results remained unchanged even when we included the recalls of those series in which the processing component had been interrupted. The mean 
Table 1

Mean Working Memory Spans (and Standard Deviations) Observed in Experiment 3 as a Function of the Type and Duration of the Task

\begin{tabular}{|c|c|c|c|c|c|c|c|}
\hline \multirow[b]{3}{*}{ Duration } & \multicolumn{6}{|c|}{ Task } & \multirow[b]{3}{*}{$M$} \\
\hline & \multicolumn{2}{|c|}{ Baba } & \multicolumn{2}{|c|}{$\begin{array}{l}\text { Reading } \\
\text { operation }\end{array}$} & \multicolumn{2}{|c|}{$\begin{array}{l}\text { Continuous } \\
\text { operation }\end{array}$} & \\
\hline & $M$ & $S D$ & $M$ & $S D$ & $M$ & $S D$ & \\
\hline Short & 3.97 & 0.83 & 3.19 & 0.96 & 2.49 & 0.86 & 3.22 \\
\hline Long & 4.01 & 0.98 & 3.61 & 1.17 & 2.50 & 1.11 & 3.38 \\
\hline$M$ & 3.99 & & 3.40 & & 2.49 & & \\
\hline
\end{tabular}

continuous operation span increased (2.59) but was still lower than the mean reading operation span $(3.41), F(1,138)=16.75, p<$ $.01, \eta^{2}=.088$, that was, in turn, lower than the baba span, which remained unchanged $(4.00), F(1,138)=8.69, p<.01, \eta^{2}=.046$. The short duration still resulted in a slightly lower mean span than the long duration (3.24 and 3.43, respectively), but this effect did not reach significance $F(1,138)=1.41, p=.24, \eta^{2}=.007$.

Thus, though the spans resulting from the three working memory tasks differed greatly in the way that we had predicted, these spans did not depend on the duration of the activity. Of course, this is not to say that the duration of the task does not have any effect on span and that prolonging the duration of treatment never results in poorer recalls. It should be remembered that we compared only two durations ( $5 \mathrm{~s}$ and $9 \mathrm{~s}$ ) and that this difference might be insufficient to detect time effects. Moreover, we used a betweensubjects design that might be insufficiently sensitive. However, we can conclude that, even if the raw duration of the processing component of working memory span tasks has some effect on span, working memory spans depend primarily and to a greater extent on the nature of the processing component.

\section{Discussion of Experiments 1-3}

This first series of experiments led us to three main conclusions. The first concerns the effect of cognitive load on working memory spans: Even when the duration of the processing component was held constant, more demanding treatments resulted in lower spans. The second conclusion concerns the nature of the concurrent tasks and thus questions the usual conceptions of the cognitive load. Abandoning self-paced activities, our new computer-driven paradigms make it clear that even fairly simple tasks such as adding and subtracting 1 or 2 or reading digits could strongly impair maintenance and recall. The third point concerns time effects that are more complex than is suggested by the task-switching hypothesis. These three points are discussed in turn.

As far as the first point is concerned, this series of experiments makes it clear that working memory spans strongly depend on the nature of the treatment participants have to perform while maintaining memory items. As Barrouillet and Camos (2001) had already observed in children, the operation span is lower than the baba span in adults when the duration of the two tasks is kept constant. The following experiments demonstrated that even when both time and the articulatory activity parameters are carefully controlled, great differences in spans still appear as a function of the cognitive cost of the task. The continuous operation span, which requires successive retrievals of arithmetic facts from longterm memory, was lower than the reading operation span that simply requires participants to read instead of solving the same operations. These results lend firm support to the general resourcesharing view of working memory span tasks and challenge Towse et al.'s (1998) task-switching hypothesis. Working memory spans do not primarily depend on the duration of the concurrent task but on the cognitive load it involves.

Second, these results not only provide evidence that the cognitive load determines working memory spans, but they also shed light on the processes that underpin cognitive load. The new working memory tasks we designed (i.e., the continuous and reading operation span) involve series of retrievals, each of which is fairly simple. However, these tasks are not subject- but computer-paced. Thus, they largely hamper strategies that participants use to suspend treatment in order to keep short pauses free for the restoration of decaying memory traces before resuming the activity. As our theory predicts, continuous and even reading operation tasks had a highly detrimental effect on span. We observed a mean (traditional) operation span of 3.49 in adults (Experiment 1), whereas samples issuing from the same population reached only 3.15 and 3.40 with the reading operation span task (Experiments 2 and 3, respectively), and fell to 2.60 and 2.49 when the participants were presented with the continuous operation span task. For the sake of comparison, Barrouillet and Camos (2001) observed an even better traditional operation span in 11-year-old children (2.83)! These findings illustrate the fact that there is no need to use complex activities such as sentence comprehension, reasoning, or problem solving to constrain recalls in working memory span tasks. As our model predicts, and as we show in the following experiments, spans do not depend on complexity but on the time during which the processing component captures attention, which is a function of both the number of retrievals:time ratio and the difficulty of these retrievals.

Thus, and this is our third point, Towse and Hitch (1995) were right in surmising that spans greatly depend on time parameters, but time effects on working memory spans are far more complex than their task-switching hypothesis suggests. Contrary to their predictions, equal durations can result in different recall performance, whereas different durations sometimes result in unchanged spans. These two phenomena are accounted for by our theory. Because working memory spans depend on the cognitive load, keeping time constant does not prevent changes in span if there are more retrievals to be performed or if they are more difficult. In the same way, prolonging the treatment can result in unchanged spans because simply making the task longer implies that the number of retrievals increases proportionally with time, thus keeping the critical ratio unchanged. Our theory accounts for these results but also permits original predictions that are tested in the following experiments.

This second series of three experiments focused on two specific predictions about the time-related effects within the time-based resource-sharing theory. First, increasing the time during which attention is captured by a task while maintaining its raw duration constant should result in a higher cognitive load. Thus, increasing the number of retrievals that the processing component requires while keeping the total time constant should increase the cognitive load and thus result in lower spans. This first prediction was tested 
in Experiment 4, in which the processing component of the working memory task consisted of reading series of numbers while maintaining letters. Second, reducing the total duration of an activity that remains unchanged should increase its cognitive load. Thus, reducing the time allowed to perform a constant number of retrievals from memory should also increase the cognitive load and thus result in lower spans. This prediction was tested in two experiments. In Experiment 5, we manipulated the time allowed to read a constant number of digits while maintaining letters, whereas in Experiment 6 we manipulated the total time allowed to perform continuous operations in which the number of sign-operand pairs was kept constant. Our prediction in these last two experiments was that shorter total durations of processing should result in lower spans. In other words, working memory span should depend on retrieval pace: the faster this pace, the lower the spans.

\section{Experiment 4}

Increasing the number of retrievals while keeping time constant should result in a higher cognitive load and thus in lower spans. Experiment 4 tested this hypothesis using a working memory span task in which adults were asked to maintain letters in memory while reading aloud series of numbers presented on screen at either a fast or a slow pace. After the presentation of each letter, either 10 or 6 numbers were successively displayed on screen at a regular rhythm over a total period of $6 \mathrm{~s}$. According to Equation 2, performing a larger number of retrievals (10 instead of 6) over a fixed period of time should result in a higher cognitive load. Thus, we predicted lower working memory span in the first, fast-paced, condition than in the second, slow-paced, one.

\section{Method}

Participants. Forty undergraduate psychology students at the Université de Bourgogne received a partial course credit for participating. None of them had taken part in the previous experiments. They were randomly assigned to either the fast- or the slow-paced groups.

Material and procedure. As in the previous experiments, participants were presented with series of consonants of ascending length (from one to seven) that were presented in the same way as in Experiment 2. After each letter, either 6 or 10 numbers for the slow- and fast-paced conditions, respectively, were randomly selected from the numbers from 1 to 12 and successively displayed on screen. In the fast-paced condition, each number appeared for $450 \mathrm{~ms}$ after a delay of $150 \mathrm{~ms}$ (total of $600 \mathrm{~ms}$ ), whereas the corresponding durations for presentation and delay were 700 and $300 \mathrm{~ms}$, respectively, for the slow-paced condition (total of 1,000 ms). Thus, in both conditions, the duration of the interletter intervals was the same (i.e., $6 \mathrm{~s}$ ). The participants were asked to read aloud all the letters and numbers that appeared on screen.

At the end of each series of letters, the word Rappel was displayed on screen to inform the participants that they had to recall the letters in their order of appearance. As in the previous experiments, the task was interrupted as soon as the participants failed to correctly recall the letters in each set of a given length series. The spans were calculated as in Experiment 1.

\section{Results}

In both conditions, the number reading task was seldom interrupted (only one and three interruptions in the slow and fast conditions, respectively), suggesting that participants paid sufficient attention to this activity. Thus, all the recalls were taken into account even after an interruption of the concurrent task. As we predicted, the number of retrievals to be performed after each letter had a strong effect on span. The mean span in the slow-paced condition was $4.28(S D=1.01)$, whereas it dropped to $2.77(S D=$ $0.92)$ in the fast-paced condition, $t(38)=4.95, p<.01, \eta^{2}=.392$.

Though the concurrent task to be performed in this experiment was, at first glance, a very simple one (i.e., reading small numbers), it is worth noting that the fast-paced condition resulted in a particularly low mean working memory span (2.77). This result lends considerable support to our theory, which suggests that the cognitive load depends mainly on the pace at which retrievals from memory have to be performed. Actually, working memory spans increased substantially when this pace was reduced, even when the total duration of retention was held constant. However, increasing the number of retrievals is not the only way to increase the pace of retrieval and thus the cognitive load. According to Equation 2, comparable changes in cognitive load should be obtained by reducing the time allowed to perform an unchanged number of retrievals from memory. Experiments 5 and 6 focused on this prediction.

\section{Experiment 5}

Experiment 4 demonstrated that increasing the number of retrievals:time ratio by increasing the number of retrievals to be performed resulted in lower spans. In the present experiment, this ratio was increased by decreasing the time allowed to perform a constant number of retrievals. According to Equation 2, any reduction in the time $T$ allowed to perform a constant number $N$ of retrievals should result in a higher cognitive load and consequently in lower working memory spans. The present experiment tested this hypothesis using the same number reading span task as in the previous experiment. Two groups of adults were presented with series of letters to be remembered and numbers to be read in the interletter intervals. We manipulated the time available to read these numbers while keeping the volume of numbers constant. In the slow-paced condition, participants were given $1 \mathrm{~s}$ to read each number, whereas in the fast-paced condition, this time was reduced to $600 \mathrm{~ms}$. We predicted that the latter condition would result in a higher cognitive load and thus in lower working memory spans. It should be noted that Towse et al.'s (1998) task-switching hypothesis makes exactly the opposite prediction. Because a faster presentation shortens the total duration of the concurrent task, their task-switching model assumes that the retention periods are reduced, thus resulting in better recalls and higher working memory spans.

\section{Method}

Participants. Forty-eight undergraduate psychology students at the Université de Bourgogne received a partial course credit for participating. None of them had taken part in the previous experiments. They were randomly assigned to either the fast- or the slow-paced groups.

Material and procedure. The material and procedure were the same as in the previous experiment. However, in Experiment 5, the numbers to be read were only one-digit numbers. The participants were presented with series of consonants of ascending length (from 1 to 7 ) with 3 series of each length (i.e., a total of 84 letters), and we thus created 84 series of 5, 6, or 7 digits. Both the fast- and slow-paced groups saw exactly the same series of letters and digits. The only difference was the pace at which the digits 
were presented. As in Experiment 4, the overall time allowed to read each digit was $600 \mathrm{~ms}$ in the fast-paced group and 1,000 ms in the slow-paced group. The task was interrupted and the spans calculated using the same procedure as in the previous experiments.

\section{Results}

As in the previous experiment, the rate of interruption of the reading digit task was very low in both groups (i.e., no interruptions in the slow condition and $1 \%$ of the presented series in the fast condition). Thus, all the recalls were included in the analyses. As we predicted, the fast-paced presentation of digits resulted in a lower mean working memory span $(3.01, S D=0.62)$ than the slow-paced presentation $(4.67, S D=0.88), t(46)=7.54, p<.01$, $\eta^{2}=.553$. Thus, the faster the pace at which successive retrievals have to be performed, the more difficult the maintenance of the memory items and the poorer the recall performance. This result provides strong support for our theory and rules out the taskswitching model that predicts that intervening treatments of shorter duration will result in better recalls. Before discussing this striking result, we present an attempt to replicate and extend it using another working memory task that involved different retrievals from long-term memory.

\section{Experiment 6}

This experiment contrasted two groups that performed a continuous operation span task in which we manipulated the pace of presentation of the sign-operand pairs. More precisely, each continuous operation involved four sign-operand pairs, but each of these pairs was presented for either $1.2 \mathrm{~s}$ in the fast-paced condition or $2.0 \mathrm{~s}$ in the slow-paced condition. As in Experiment 5, we predicted lower working memory spans in the fast-paced condition than in the slow-paced condition.

\section{Method}

Participants. Forty undergraduate psychology students at the Université de Bourgogne received a partial course credit for participating. None of them had taken part in the previous experiments. They were randomly assigned to either the fast- or the slow-paced groups.

Material and procedure. The material and procedure were the same as in the continuous operation span presented in Experiment 2. The only difference was that each continuous operation involved four sign-operand pairs that remained on screen for either $1.2 \mathrm{~s}$ or $2.0 \mathrm{~s}$.

\section{Results}

The continuous operation task proved to be extremely difficult for adults when presented at a fast pace. The rate of interruption of the continuous operations was $12 \%$ in the fast-paced group compared to $2 \%$ in the slow-paced group. These interruptions concerned $23 \%$ and $5 \%$ respectively of the series of letters presented and recalled. This high rate of interruption made it impossible to omit the series concerned from the analyses. Thus, though frequent interruptions of the intervening treatment could facilitate strategies for the rehearsal or refreshment of memory traces, all the recorded recalls were taken into account, even for series in which the concurrent continuous operation task was not correctly completed. Though taking these series into account ran counter to our hypothesis, the participants in the slow-paced group (mean span $=2.88$,
$S D=1.24)$ largely outperformed those in the fast-paced group, whose mean continuous operation span fell dramatically to 1.80 $(S D=0.70), t(38)=3.41, p<.01, \eta^{2}=.234$. Thus, these results wholly confirmed our previous observations. In line with the predictions issuing from our theory, any reduction in the time allowed to perform a given task increases its cognitive cost.

\section{Discussion of Experiments 4-6}

The present series of experiments tested the hypothesis that cognitive load depends on the proportion of time during which attention is captured. For tasks that mainly involve retrievals from memory, cognitive load should be a function of the number of retrievals:time ratio. For a given period of time, increasing the number of retrievals should result in shorter interretrieval pauses during which memory traces can be refreshed and restored (Experiment 4). The same results should also be obtained by reducing the total time available to perform a constant number of retrievals (Experiments 5 and 6). In all of these experiments, a higher number of retrievals:time ratio resulted in poorer recalls and lower working memory spans, even when the total duration of the intervening treatment was reduced (Experiments 5 and 6). This last result definitely rules out Towse and Hitch's (1995) task-switching model: Shorter treatments can lead to lower working memory spans. In accordance with our model, this fact suggests that participants switch their attention from processing to maintenance during the intervening activity and that the relative difficulty of this switching process determines what is called cognitive load.

Moreover, the results of this second series of experiments provide strong support for our theory by making it clear that even very simple tasks can have a highly detrimental effect on recall when included in working memory span tasks. When compared with a traditional operation span such as that used in Experiment 1 (mean span of 3.49), reading digits at a moderate pace of one digit per second resulted in high working memory spans (4.28 and 4.67 in Experiments 4 and 5, respectively). However, a simple reduction in the time available to read these digits (600 ms instead of $1 \mathrm{~s})$ resulted in a major decrease in the mean spans, which dropped below the level of the mean operation span (2.77 and 3.01, respectively).

Thus, this second series of experiments confirmed the main predictions issuing from our time-based resource-sharing model. Both treatment and storage activities compete for a unique spotlight of retrieval while memory traces suffer from a time-related decay. As a consequence, time-related parameters such as the pace at which the treatment is completed become crucial because they determine the possibility of switching attention away to refresh and restore decaying memory traces. However, the previous experiments involved comparisons between pairs of conditions and did not allow us to test the prediction issuing from Equation 2 of a linear function between cognitive load and the number of retrievals:time ratio. This hypothesis was tested in Experiment 7.

\section{Experiment 7}

The resource-sharing hypothesis implies that there is a trade-off between processing and storage. However, our theory goes beyond the prediction that increasing the cognitive load of the processing component results in poorer recall, as Experiments 4-6 demon- 
strated, by predicting the shape of the function that relates processing and storage. Our theory assumes that cognitive load is a linear function of the number of retrievals:time ratio. Progressively increasing this ratio should result in a smooth and linear decrease in span. Moreover, because cognitive load is also a function of the difficulty of these retrievals (parameter $a$ ), the decrease in span should be all the more pronounced the more difficult the retrievals are. We tested these hypotheses by presenting nine groups of adult participants with nine different values of the ratio (from 0.4 to 2.0) in a reading digit span task. The specific effect of difficulty was tested in three additional groups of adults who performed a $b a b a$ span task at three of the nine ratios $(0.4,1.0$, and 2.0). We predicted that, for both tasks, the recall performance would decrease as the number of retrievals:time ratio in the processing component increased and that this decrease would be less pronounced in the baba condition than in the reading digit condition because, in the former, the participants simply have to keep track of a habituated stimulus and always produce the same response.

\section{Method}

Participants. Twelve groups of 16 undergraduate psychology students at the Université de Bourgogne received a partial course credit for participating. None of them had taken part in the previous experiments. Nine groups performed a reading digit span task, and three groups performed a baba span task.

Material and procedure. The reading digit span task was essentially the same as in Experiment 4. The consonants to be recalled were successively displayed on screen in series of increasing length (from one to eight) with three series of each length. Within each series of letters, a series of 4 , 8 , or 12 numerals (from 1 to 12 ) in their Arabic form was displayed on screen after each letter for a total period of $6 \mathrm{~s}, 8 \mathrm{~s}$, or $10 \mathrm{~s}$, thus resulting in nine different values of the number of retrievals:time ratio (from 0.4 to 2.0). The numerals appeared at a constant pace that was determined by dividing the interval between two successive letters (either $6 \mathrm{~s}, 8 \mathrm{~s}$, or $10 \mathrm{~s}$ ) by the number of numerals to be presented $(4,8$, or 10$)$. Each numeral remained on screen for $75 \%$ of the resulting duration after a blank screen lasting for $25 \%$ of this duration. For example, for 12 numerals in $10 \mathrm{~s}$ mode, each numeral remained on screen for $625 \mathrm{~ms}$ after a delay of $209 \mathrm{~ms}$. The participants were instructed to read these numerals aloud and to recall the letters of the series when the word Recall appeared. The same design was used for the $b a b a$ span task except that it involved only the three ratio values of $0.4,1.0$, and $2.0(4,8$, and 12 syllables presented, respectively, over $10 \mathrm{~s}, 8 \mathrm{~s}$, and $6 \mathrm{~s}$ ), and that the syllable $b a$ appeared repeatedly on screen instead of numerals. To facilitate discrimination between successive presentations, we presented the syllable $b a$ in small and capital letters alternately. The same series of letters was presented in each experimental condition for both tasks. In both tasks, the participants were presented with three 1-letter and three 2-letter training series.

\section{Results}

Concerning the reading digit span task, a 3 (numbers of numerals: 4,8 , or 12) $\times 3$ (time: $6 \mathrm{~s}, 8 \mathrm{~s}$, or $10 \mathrm{~s}$ ) analysis of variance on the mean spans revealed that, as our theory predicts, the spans decreased as the number of numerals increased (4.90, 4.00, and 3.41 for 4,8 , and 12 numerals, respectively), $F(2,135)=23.33$, $p<.01, \eta^{2}=.227$, and that shorter durations resulted in lower spans $(4.55,4.19$, and 3.56 for $10 \mathrm{~s}, 8 \mathrm{~s}$, and $6 \mathrm{~s}$, respectively), $F(2$, $135)=10.40, p<.01, \eta^{2}=.101$, without any interaction between the two factors, $F<1$. More important, a linear regression revealed that recall performance was highly correlated with the number of retrievals:time ratio $(r=-.965)$, which accounted for more than $93 \%$ of the variance in mean spans (see Figure 2). Concerning the $b a b a$ span, varying the rate of pronunciation of $b a$ induced a decrease in mean spans $(5.27,4.94$, and 4.08 for rates $0.4,1.0$, and 2.0, respectively), $F(2,45)=3.13, p=.05, \eta^{2}=$ .122. As we predicted, this effect was smaller than that observed with numerals $(5.46,4.25$, and 2.67 , respectively), as testified by the significant interaction between tasks and ratios, $F(2,90)=$ 3.38, $p<.05, \eta^{2}=.047$, and reading numerals resulted in significantly lower spans than repeating the syllable $b a, F(1,90)=$ $6.42, p<.05, \eta^{2}=.044$. The intercepts of both regression lines were very close, but the slopes differed greatly $(-0.75$ and -1.49 , respectively; see Figure 2).

Moreover, our theory not only predicts the observed relationship between the cognitive demand of processing and recall performance, but it also predicts that the intercepts of the regression line should correspond to performance in both components when performed independently. This was indeed precisely what we observed. On the one hand, both regression lines approximately converged on the same intercept on the $y$-axis (5.66 and 5.62 for reading numerals and concurrent articulation, respectively). This span value, which corresponds to a recall performance without any secondary task, results in our experiment from the correct recall of at least two out of the three series of six letters. Accordingly, this performance corresponds to the mean adult letter span usually observed in short-term memory studies, that is to say six (Dempster, 1981). On the other hand, the extrapolation of the regression line for numerals gives an intercept on the $x$-axis of 3.80 , that is a rate of 3.80 numerals read per second. This value should correspond to an optimal performance in reading digits when there is no concurrent memory load. Interestingly, it is very close to the

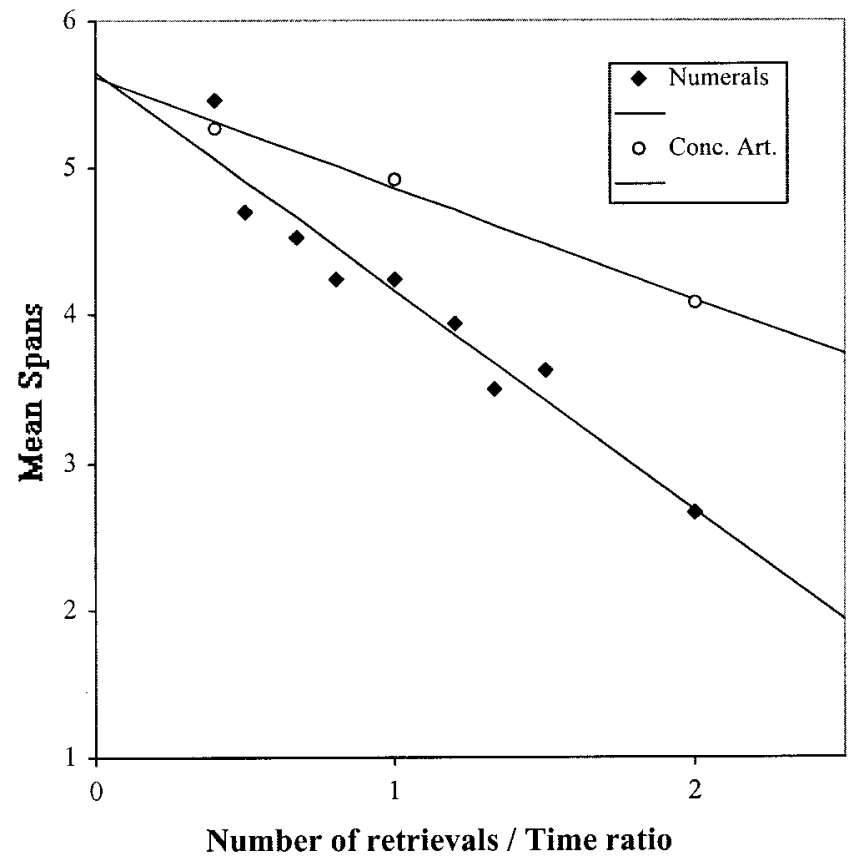

Figure 2. Mean spans per group as a function of the number of retrievals: time ratio and the nature of the processing component (reading numerals or saying "baba"). Conc. Art. = concurrent articulation. 
maximum reading rate for digits reported by Naveh-Benjamin and Ayres (1986), that is 3.91 for languages with one-syllable digits, which is the case for French. Thus, as predicted by the resourcesharing hypothesis, the trade-off function connects the maximum performances that could be achieved on both tasks if they were performed independently in the most favorable conditions.

\section{Discussion}

Overall, our results clearly indicated that when time parameters are controlled for, there is a quasi perfect trade-off between processing and storage. The linear trends confirmed that the cognitive demand of a task corresponds to (a) the difficulty of the retrievals it requires and (b) the number of these retrievals divided by the time allowed to perform them. It should be noted that the relationship we observed between the critical ratio and the recall performance confirms one of the main assumptions of any capacity theory; namely, that mental effort consumes some kind of mental energy and that certain activities consume more energy than others. The present experiment extends this physical analogy by demonstrating that the cognitive demand or mental effort involved in a cognitive activity conforms to the physical law of power: It is a function of the amount of work this activity requires divided by the time taken to do it. The cognitive load that a task involves corresponds to the mental power needed to perform it, that is, the rate at which mental energy is converted. Thus, working memory spans do not depend on the duration of the processing component but on the mental power it requires, that is, the rate at which it has to be performed.

\section{General Discussion}

All the experiments reported in this article confirmed the two main aspects of our model. First, working memory span tasks involve a resource-sharing process between treatment and storage. As we predicted, working memory spans vary as a function of the nature of the treatment, even when total duration is kept constant. Second, the sharing of resources between processing and storage is a time-constrained process: Working memory spans depend on the pace at which the processing component has to be performed, any series of very simple retrievals being sufficient to capture attention and to have a detrimental effect on recall. As we stressed previously, these facts lend strong support to the main assumptions on which our theory is based. First, processing components within working memory span tasks do not require complexity to disrupt maintenance. Simple activities that rely on the memory retrieval process, which is also needed to refresh decaying memory traces, can have a highly detrimental effect on spans. Second, this common process implies a time-based resource sharing between activities because retrieval constitutes a structural bottleneck. Thus, a rapid switching mechanism is needed between processing and storage. Third, this switching is time-constrained because memory traces suffer from a time-related decay. Finally, our results make it possible to clarify the nature of working memory limitations. These four topics are addressed in turn.

\section{Retrieval, Activation, and Attention}

As pointed out by Baddeley (2000), the concept of working memory has been developed to emphasize the functional role of short-term memory in maintaining, but also in manipulating, information during the completion of complex cognitive tasks. Thus, multicomponent (Baddeley \& Hitch, 1974) as well as unitary models of working memory (Case, 1985) have made a clear distinction between systems or spaces devoted to executive control, on the one hand, and passive maintenance, on the other. This sharp distinction seems to have had two main consequences. The first concerns the way working memory span tasks have been conceived. The second concerns the theoretical accounts that have been proposed for the relationship between processing and storage in working memory span tasks and, more generally, for the role of working memory in complex cognitive activities.

As far as the first point is concerned, the tasks that are intended to evaluate working memory capacities have usually been designed to reflect the heterogeneity between active processing and passive storage. Complex span tasks were conceived that involve a traditional memory span task, such as a word or a digit span, coupled with an intervening and disruptive activity intended to tap the executive control component (Daneman \& Carpenter, 1980; Turner \& Engle, 1989). In consequence, these activities were selected among those supposed to require a high level of executive control (e.g., problem solving, reading comprehension, reasoning, mental calculation), the underlying idea being that the more controlled and complex the activity, the better the working memory task.

Second, the postulate of a sharp difference between storage and executive control has probably led researchers to overlook the functional aspects of the simultaneous completion of both components in working memory span tasks. The limited resource for which processing and storage are thought to compete has often been described in somewhat vague terms, such as "cognitive space" (Case, 1985) or "mental power" (Pascual-Leone, 1970, 1978). Moreover, the dissimilarity between processing and storage has led authors to situate this shared resource in higher order processes such as controlled attention (Engle et al., 1999; Kane, Bleckley, Conway, \& Engle, 2001) or capacities for coordinating multiple-system functioning (Baddeley, 1990). Accordingly, it is generally assumed that the construct termed "working memory" is especially suited to account for high-level cognitive processes involved in complex tasks (Daneman \& Hannon, 2001; Shah \& Miyake, 1999).

In contrast to this approach, our theory assumes that processing and storage compete for attention that is needed to perform the processing component of the task but also to frequently refresh the decaying memory traces through a retrieval process. In consequence, we predicted that complex activities were not needed to disrupt the maintenance of memory items. Provided that they capture attention for frequent and sufficiently long periods of time, even simple tasks were expected to have a detrimental effect on working memory span. For example, tasks that constrain participants to perform series of retrievals from long-term memory at a fixed rate were expected to have a highly detrimental effect on maintenance because these retrievals totally capture attention due to a central bottleneck. In fact, our experiments demonstrated that they have a more detrimental effect on recall than complex but self-paced activities such as problem solving. This fact suggests that the self-paced character of the main working memory span tasks used in the literature has led researchers to overestimate, and probably misunderstand, the relationship between the complexity 
of the processing component and the observed spans. Two main conclusions can be derived from this finding. First, time parameters are more important than complexity. This point is addressed in the following section. Second, very simple retrievals do indeed tap some kind of limited resource, which is also needed to maintain memory items. This fact lends strong support to those models of working memory that conceive of resources as a kind of mental energy available for activation (Anderson, 1993; Anderson \& Lebiere, 1998; Just \& Carpenter, 1992; Lovett et al., 1999).

To conclude, the present study introduces a new conception of cognitive cost for which we gathered ample evidence and that departs from the idea of complexity. First, simple retrievals involve a high cognitive cost, provided that we still consider that impairments to concurrent maintenance and subsequent recall are indicative of cognitive cost. Second, reducing the number of retrievals a given task requires should lead to a decrease in the cognitive cost it involves. This is in line with Logan (1988), who suggested considering automatization as a shift from algorithmic processing to a single direct memory retrieval. According to our model, algorithms are demanding because they require multistep processing and successive retrievals from long-term memory (Barrouillet \& Fayol, 1998; Siegler, 1996). The shift from algorithmic processing to the direct retrieval of the answer from memory would thus correspond to a decrease in the number of retrievals the task requires, that is, a decrease in the cognitive load. Finally, any task that requires memory retrievals can become a demanding one if it is presented at a fast pace. It can be assumed that tasks that do not involve memory retrievals but capture attention should also have an effect on span all the more detrimental as the pace at which they are performed increases. What remains an open question is whether tasks that require retrievals involve a higher cognitive load than tasks that occupy central processing for equivalent periods of time by other processes (e.g., selection of response). This problem requires further studies. Nonetheless, as we have emphasized previously, time parameters are more important than complexity. We now turn to this point.

\section{Time Constraints and the Switching Process}

As we pointed out above, Towse and Hitch (1995) were right in suggesting that working memory spans mainly depend on time parameters because working memory tasks require participants to switch attention from storage to processing, whereas memory traces suffer from a time-related decay. Moreover, they were right in surmising that the spans do not entirely depend on the nature of the secondary task. However, as we have seen, the relevant factor is not, as they assumed, the entire duration of the processing part of the task. All of our results contradicted this hypothesis. The first series of experiments showed that working memory spans can vary even when the duration of the task is kept constant (Experiments 1 and 2, but also 5) and that prolonging the processing component of the task does not necessarily result in lower spans (Experiment 3). More important, increasing the time allowed to perform an unchanged number of retrievals resulted in even better recalls (Experiments 5, 6, and 7). Finally, Experiment 7 deciphered the relationship between durations and spans: All other things being equal, the shorter the task, the lower the span! This body of empirical facts leads to the compelling conclusion that individuals manage to perform processing and storage "simultaneously."
Thus, contrary to what Towse and Houston-Price (2001) suggested, the idea of limited resource-sharing capacity is not superfluous in understanding cognitive processes. We can still consider that some activities involve a greater cognitive load and consume more resources than others because they have a more detrimental effect on a concurrent activity performed simultaneously. These activities are probably experienced as more difficult and tiring. Our results suggest that, exactly like physical effort, mental effort is a function of the amount of work to be done divided by the time allowed to do it. Working memory spans are a direct function of the mental effort the processing component requires.

However, this does not mean that apparently simultaneous treatments imply the sharing of a continuously divisible resource between two tasks that are then processed absolutely in parallel. If such continuous resource sharing were involved in working memory span tasks, a concurrent activity as simple as reading digits should have a minor impact on spans, whereas solving complex arithmetic problems should have a stronger impact. Indeed, the former activity should leave a large amount of unoccupied resources that would be available for keeping the memory traces active, whereas the latter should capture a larger part of this divisible resource and thus result in poorer recalls. This is not what we observed.

Instead, the strong impact that successive simple retrievals had on working memory spans suggests, in line with Pashler's proposal (1998; Carrier \& Pashler, 1995), that memory retrieval is subject to a discrete processing bottleneck that prevents simultaneous retrievals. Thus, until participants have finished retrieving the phonological representation of a given digit, they cannot start remembering the series of letters to be recalled to update their memory traces. In the same way, Garavan (1998) argued that people are able to attend to just one object at any one time and that many cognitive tasks necessitate switching between memory items. Therefore, the decaying activation of the letters could only be restored during the interretrieval intervals by means of a rapid switching process. The hypothesis of a central bottleneck for retrieval accounts perfectly for the fact that working memory spans were lower when the duration of the interdigit intervals was reduced, either by reducing the total time allowed to read the digits or by increasing their number. This phenomenon echoes previous observations reported by Pashler (1998) from Kalsbeek and colleagues who showed that, in dual-task experiments, performance on a secondary task depended on the rate at which the primary task was presented (Kalsbeek \& Sykes, 1967; Schouten, Kalsbeek, \& Leopold, 1960). This effect of the rate at which the secondary task is performed suggests that what we can still describe, at a macroanalysis level, as resource sharing (more difficult processing components result in lower spans) appears to be a time-sharing phenomenon at a more atomic level.

Moreover, our theory also accounts for the fact that traditional working memory span tasks such as the operation span task (Turner \& Engle, 1989) can be easier than our continuous operation span task, which simply requires small and automatized additions and subtractions. Indeed, complex activities can be interrupted for many seconds and then resumed without detectable cost (Gillie \& Broadbent, 1989). Thus, when performing self-paced operation solving, individuals are free to strategically interrupt their calculation to update the list of memory items, even if arithmetic problems are difficult. In the same way, before reading 
the next letter or the "recall" signal, they can simply postpone their response on the keypad to rehearse the letters that have already been presented. All these strategies are hampered when tasks are computer paced because the activities of updating and refreshing of the memory traces are constrained and hampered by the pace at which the information to be processed enters working memory. This is probably the reason why self-paced working memory span tasks require complex activities as processing components to disrupt maintenance and recall. Complex activities involve algorithmic processing that necessitates several successive retrievals from memory as well as the temporary storage and retrieval of intermediate results. A large number of retrievals and the need to maintain intermediate results impede attentional shifts to the items to be recalled. However, complexity is not required per se: What is needed is a steady capture of attention. This can be achieved through series of retrievals in quick succession that occupy the spotlight of retrieval and prevent, although imperfectly, strategies for the rehearsal or refreshment of memory traces. While attention is switched away from the memory items, they suffer from a time-related decay.

\section{Time-Related Decay of Memory Traces}

In accordance with many models of short-term as well as working memory, we assume that short-term memory consists of the permanent knowledge that is activated above threshold (Cantor \& Engle, 1993; Cowan, 1995; Just \& Carpenter, 1992). Because activation decays spontaneously with the passage of time, refreshing processes are needed to keep this knowledge available (Baddeley, 1990; Cowan, 1995; Engle et al., 1999; Henson, 1998; Page $\&$ Norris, 1998). These refreshing processes can take the form of overt or covert rehearsal (Baddeley, 1986), but also of memory searches (Cowan et al., 1998). Because all these processes involve the retrieval of the memory traces that are temporarily outside of the focus of attention, they require attention and thus compete with those steps of the concurrent treatment that capture attention, and particularly with concurrent retrievals that block the central bottleneck. All our results confirmed this model and suggest that the shorter the periods during which refreshing processes could be used, the poorer the recalls.

However, this model of time-related decay of activation has often been challenged. For example, Nairne (1988, 1990, 2002; Neath \& Nairne, 1995) has proposed the feature model in which short-term forgetting does not result from the time-related decay of activation. Instead, it is thought to be due to item-based interference provoked by intervening events. According to the feature model, short-term memory does not consist of those activated items that can be directly retrieved but rather of a constellation of activated cues that make it possible to reconstruct what happened moments before. Previous processing records that constitute these retrieval cues are overwritten by subsequently occurring material. Thus, the available cues become poor predictors of the target items and forgetting occurs.

It should be noted that the hypothesis of a short-term memory containing retrieval cues rather than "items" of knowledge can be accommodated within our proposal. It is quite conceivable that the traces of these retrieval cues themselves fade away with the passage of time. By contrast, the assumption that there is no time-related decay of memory traces and that forgetting results from interference is clearly contrary to our conception. Some results accord more with the time-related decay hypothesis than with the interference hypothesis. Consider, for example, Experiment 5 in which participants were presented with an unchanged number of digits whose rate of presentation was, however, manipulated (one digit per $1 \mathrm{~s}$ or $600 \mathrm{~ms}$ ). In terms of the feature model, both the amount and the nature of occurring material remained exactly the same across conditions. Thus, the feature model would predict the same level of forgetting in both conditions. By contrast, the hypothesis that memory traces suffer from a time-related decay when attention is switched away in a rapid switching process predicts poorer recalls when digits are presented at a higher rate because refreshing periods are shorter. The results clearly supported our prediction, which was confirmed in Experiments 6 and 7. Nonetheless, it could be argued that when digits are presented at a slower rhythm, the letters to be remembered are presented with increased time intervals. According to the oscillator-based associative recall model (Brown, Preece, \& Hulme, 2000), these increased intervals could result in retrieval cues that are less susceptible to confusion and thus in better recall. However, if this were the case, simply prolonging the activity, as we did in Experiment 3 , should result in the same increase in correct recalls. In fact, there was no difference between short and long task durations in Experiment 3 . As predicted by our theory, simply prolonging the task left the pace unchanged and resulted in equivalent spans.

Thus, our results are in accordance with the well-established hypothesis that memory traces in short-term memory suffer from a time-related decay. Such a conception could lead to the conclusion that working memory span tasks involve time sharing rather than resource sharing and that the notion of resource is not needed to account for the phenomena involved in working memory span tasks. However, it should be remembered that working memory tasks are of interest because they provide us with span measures that predict performance in a wide range of cognitive activities. Thus, any model of working memory tasks must explain what it is that working memory span tasks really measure and how what they measure is involved in other cognitive tasks. We address these questions in the next section.

\section{Nature of Working Memory Limitations and the Working Memory Span Measures}

According to our model, at least three main factors could limit working memory functioning and consequently performance in working memory span tasks. These factors correspond to the three points discussed previously. The first is the amount of available attention that produces activation and thus constrains the retrieval process. The second concerns the switching mechanism itself, whereas the third relates to the phenomenon of decay.

Even if we abandon the hypothesis of the sharing of a continuously divisible resource, the general notion of resource remains central to our theory. According to Anderson's ACT-R theory (1993; Anderson \& Lebiere, 1998), the amount of attentional energy available to produce activation and achieve retrievals is limited. This theory predicts that people with higher attentional capacity will have a higher probability of retrieving information from memory and will perform their retrievals faster (Rosen \& Engle, 1997). Thus, these individuals will be less constrained by the time parameters that determine the cognitive cost, and they 
should have higher working memory spans. Of course, these high attentional capacities could also ensure high performance levels in a wide range of complex cognitive activities that require individuals to retrieve large amounts of knowledge from memory, as well as to maintain and quickly retrieve intermediate results. Such a factor, which links retrieval speed and working memory span, could also account for the relationship that Case et al. (1982) observed between maximum counting speed and counting span in children and adults. Faster counting results from the faster retrieval of the successive number words from the number line (Camos, Barrouillet, \& Fayol, 2001). These faster retrievals could in turn free up longer periods during which the unoccupied spotlight of retrieval could be used to refresh the memory traces of the totals already counted. In the same way, a developmental increase in the amount of available attentional energy could account for the developmental increase in working memory span (Barrouillet \& Camos, 2001). For example, Cowan et al. (1994) studied time parameters during the verbal response period of a short-term memory task. They observed that age did not affect the duration of word pronunciation in the response but affected the duration of silent periods both between the end of the list and the beginning of the response and between words during this response (interword pauses). They thus assumed that age affects the speed of covert processing such as the memory search, which is probably used to reactivate memory items during the interword pauses in the response. Faster memory searches in older children could result from a higher volume of attentional resources available for activating memory items.

The second factor that can limit performance in working memory span tasks concerns the efficiency of the switching process. Mental set shifting is often postulated as one of the three main executive functions along with updating and inhibition (Miyake et al., 2000). Though there is little evidence to support the assumption of a specific component of the central executive that is exclusively specialized in attention switching (Allport, Styles, \& Hsieh, 1994), Baddeley, Chincotta, and Adlam (2001) have suggested that the central executive is involved in the switching process. This is in line with Baddeley's (1990) previous assumptions about the similarity between the central executive and the supervisory attentional system proposed by Norman and Shallice (1986), which is obviously needed if participants are to simultaneously perform two tasks in unusual situations such as laboratory working memory experiments. Thus, the need to perform frequent task switching under the control of the central executive should act as a strong limitation on working memory functioning because the central executive is usually assumed to have a limited capacity (Baddeley \& Logie, 1999; Cowan, 1995; Engle et al., 1999). For the moment, empirical evidence for this hypothesized relationship is still lacking. Miyake et al. (2000) observed that controlling for updating and inhibition capacities, individuals with better attention-switching abilities did not have higher operation spans. However, the tasks used by the authors to measure switching capacities were in fact poorly correlated with all the tasks that assessed other executive functions (the highest $r$ was .26). Thus, further studies are needed to firmly establish the impact of switching efficiency on working memory spans. If individual differences in working memory span are at least in part due to differences in the switching process, working memory span should not only reflect capacities for activation and retrieval but also capacities for controlled or executive attention, as argued by Engle (2001; Kane et al., 2001).

Finally, the third limitation is due to the decay phenomenon. As we have seen, the assumption that activation suffers from a timerelated decay is central to our account of performance in working memory span tasks. It has already been assumed that phonological traces disappear after a period of $2 \mathrm{~s}$ within the phonological loop (Baddeley, 1990). Although we are not committed to such strong assumptions, we agree with Cowan (1995) that there is a time limit in short-term memory. Interindividual differences in the rate of decay could induce differences in working memory span. Faster decay would result in a sharper decrease in activation over time, and thus in poorer recalls because less strongly activated memory traces would become more difficult to retrieve. Once again, it is possible that the speed of decay changes with age.

It should be noted that these three limitations (attentional resources, switching mechanism, and memory decay) all involve a temporal dimension. Attentional resources directly constrain retrieval times (Anderson, 1993), whereas the executive processes involved in task switching are time constrained (Rubinstein, Meyer, \& Evans, 2001). Of course, the decay phenomenon is a direct function of time. This is the reason why working memory spans depend to so great an extent on time parameters that have up to now been overlooked.

\section{Concluding Comments}

Though our series of experiments were conclusive, it should be remembered that the present study has two main limitations. First, we focused exclusively on verbal working memory and verbal tasks that are mainly used to assess working memory capacity. Further studies that would extend our account to visuospatial concurrent tasks are needed because it has been argued that verbal and visuospatial working memory capacities could be distinct (Shah \& Miyake, 1996). In the same way, the effects on concurrent retrievals of the "psychological refractory period" or the serial choice-reaction time task that have been documented by Pashler and colleagues (Carrier \& Pashler, 1995; Rohrer \& Pashler, 2003) suggest that the effect on working memory spans of concurrent tasks that do not rely on retrievals but involve central processing needs to be investigated. The fact that temporal density is also the main determinant of the effect of central processing on retrieval (Rohrer \& Pashler, 2003) suggests that our account of cognitive load in terms of temporal constraints can be extended to a larger range of cognitive activities. Second, we only tested a reduced version of the equation for cognitive load where all the retrievals involve the same level of difficulty. However, our model predicts that the cognitive load increases with the difficulty of retrievals (parameter $a$ in Equation 1). Though this prediction was indirectly tested in Experiment 7 as well as in Experiment 2, in which retrieving answers of additive problems was more difficult than just reading these answers and resulted in lower spans, the specific effect of the level of difficulty of retrieval will have to be more accurately and directly tested in forthcoming experiments. Thus, even if our model of cognitive cost did succeed in predicting many of the phenomena involved in working memory span tasks, it remains only a first step toward a fuller understanding of the functioning of working memory and its constraints. 


\section{References}

Allport, A., Styles, E. A., \& Hsieh, S. (1994). Shifting attentional set: Exploring the dynamic control of tasks. In C. Umilta \& M. Moscovitch (Eds.), Attention and performance XV: Conscious and nonconscious information processing (pp. 421-452). Cambridge, MA: MIT Press.

Anderson, J. R. (1993). Rules of the mind. Hillsdale, NJ: Erlbaum.

Anderson, J. R., \& Lebiere, C. (1998). Atomic components of thought. Hillsdale, NJ: Erlbaum.

Aschcraft, M. H., \& Battaglia, J. (1978). Cognitive arithmetics: Evidence for retrieval and decision processes in mental addition. Journal of Experimental Psychology: Human Learning and Memory, 4, 527-538.

Baddeley, A. D. (1986). Working memory. Oxford, England: Clarendon Press.

Baddeley, A. D. (1990). Human memory: Theory and practice. Hillsdale, NJ: Erlbaum.

Baddeley, A. D. (1996). Exploring the central executive. Quarterly Journal of Experimental Psychology, 49(A), 5-28.

Baddeley, A. D. (2000). Short-term and working memory. In E. Tulving \& F. I. M. Craik (Eds.), The Oxford handbook of memory (pp. 77-92). Oxford, England: Oxford University Press.

Baddeley, A. D., Chincotta, D., \& Adlam, A. (2001). Working memory and the control of action: Evidence from task switching. Journal of Experimental Psychology: General, 130, 641-657.

Baddeley, A. D., \& Hitch, G. (1974). Working memory. In G. A. Bower (Ed.), Recent advances in learning and motivation (Vol. 8, pp. 647667). New York: Academic Press

Baddeley, A. D., \& Logie, R. H. (1999). Working memory: The multiplecomponent model. In A. Miyake \& P. Shah (Eds.), Models of working memory: Mechanisms of active maintenance and executive control (pp. 28 -61). Cambridge, England: Cambridge University Press.

Barrouillet, P. (1996). Transitive inferences from set-inclusion relations and working memory. Journal of Experimental Psychology: Learning, Memory, and Cognition, 22, 1408-1422.

Barrouillet, P., \& Camos, V. (2001). Developmental increase in working memory span: Resource sharing or temporal decay? Journal of Memory and Language, 45, 1-20.

Barrouillet, P., \& Fayol, M. (1998). From algorithmic computing to direct retrieval: Evidence from number and alphabetic arithmetic in children and adults. Memory \& Cognition, 26, 355-368.

Brown, G. D. A., Preece, T., \& Hulme, C. (2000). Oscillator-based memory for order. Psychological Review, 107, 127-181.

Camos, V., Barrouillet, P., \& Fayol, M. (2001). Does the coordination of verbal and motor information explain the development of counting in children? Journal of Experimental Child Psychology, 78, 240-262.

Cantor, J., \& Engle, R. W. (1993). Working-memory capacity as long-term memory activation: An individual-differences approach. Journal of Experimental Psychology: Learning, Memory, and Cognition, 25, 11011114.

Carrier, L. M., \& Pashler, H. (1995). Attentional limits in memory retrieval. Journal of Experimental Psychology: Learning, Memory, and Cognition, 21, 1339-1348.

Case, R. (1985). Intellectual development: Birth to adulthood. New York: Academic Press.

Case, R., Kurland, M., \& Goldberg, J. (1982). Operational efficiency and the growth of short-term memory. Journal of Experimental Child Psychology, 33, 386-404.

Conway, A. R. A., \& Engle, R. W. (1994). Working memory and retrieval: A resource-dependent inhibition model. Journal of Experimental Psychology: General, 123, 354-373.

Cowan, N. (1992). Verbal memory span and the timing of spoken recall. Journal of Memory and Language, 31, 668-684.

Cowan, N. (1995). Attention and memory: An integrated framework. New York: Oxford University Press.

Cowan, N. (1999). An embedded-process model of working memory. In A.
Miyake \& P. Shah (Eds.), Models of working memory: Mechanisms of active maintenance and executive control (pp. 62-101). Cambridge, England: Cambridge University Press.

Cowan, N., Keller, T. A., Hulme, C., Roodenrys, S., McDougall, S., \& Rack, J. (1994). Verbal memory span in children: Speech timing clues to the mechanisms underlying age and word length effects. Journal of Memory and Language, 33, 234-250.

Cowan, N., Wood, N. L., Wood, P. K., Keller, T. A., Nugent, L. D., \& Keller, C. V. (1998). Two separate verbal processing rates contributing to short-term memory span. Journal of Experimental Psychology: General, 127, 141-160.

Daneman, M., \& Carpenter, P. A. (1980). Individual differences in working memory and reading. Journal of Verbal Learning and Verbal Behavior, 19, 450-466.

Daneman, M., \& Carpenter, P. A. (1983). Individual differences in integrating information between and within sentences. Journal of Experimental Psychology: Learning, Memory, and Cognition, 9, 561-584.

Daneman, M., \& Hannon, B. (2001). Using working memory theory to investigate the construct validity of multiple-choice reading comprehension test such as SAT. Journal of Experimental Psychology: General, 130, 208-223.

Dempster, F. N. (1981). Memory span: Sources of individual and developmental differences. Psychological Bulletin, 89, 63-100.

De Rammelaere, S., Stuyven, E., \& Vandierendonck, A. (1999). The contribution of working memory resources in the verification of simple mental arithmetic sums. Psychological Research, 62, 72-77.

De Rammelaere, S., Stuyven, E., \& Vandierendonck, A. (2001). Verifying simple arithmetic sums and products: Are the phonological loop and the central executive involved? Memory \& Cognition, 29, 267-273.

Engle, R. W. (2001). What is working memory capacity? In H. L. Roediger, J. S. Nairne, I. Neath, \& A. M. Surprenant (Eds.), The nature of remembering: Essays in honor of Robert G. Crowder (pp. 297-314). Washington, DC: American Psychological Association.

Engle, R. W., Kane, M. J., \& Tuholski, S. W. (1999). Individual differences in working memory capacity and what they tell us about controlled attention, general fluid intelligence, and functions of the prefrontal cortex. In A. Miyake \& P. Shah (Eds.), Models of working memory: Mechanisms of active maintenance and executive control (pp. 102-134). Cambridge, England: Cambridge University Press.

Engle, R. W., \& Oransky, N. (1999). The evolution from short-term memory to working memory: Multi-store to dynamic models of temporary storage. In R. J. Sternberg (Ed.), The nature of cognition (pp. 515-556). Cambridge, MA: MIT Press.

Garavan, H. (1998). Serial attention within working memory. Memory \& Cognition, 26, 263-276.

Gillie, T., \& Broadbent, D. E. (1989). What makes interruptions disruptive? A study of length, similarity, and complexity. Psychological Research, 50, 243-250.

Henson, R. N. A. (1998). Short-term memory for serial order: The startend model. Cognitive Psychology, 36, 73-137.

Hitch, G., Towse, J. N., \& Hutton, U. (2001). What limits children's working memory span? Theoretical accounts and applications for scholastic development. Journal of Experimental Psychology: General, 130, 184-198.

Just, M. A., \& Carpenter, P. A. (1992). A capacity theory of comprehension: Individual differences in working memory. Psychological Review, 99, 122-149.

Kalsbeek, J. W. H., \& Sykes, R. N. (1967). Objective measurement of mental load. Acta Psychologica, 27, 253-261.

Kane, M. J., Bleckley, M. K., Conway, A. R. A., \& Engle, R. W. (2001). A controlled-attention view of working memory capacity. Journal of Experimental Psychology: General, 130, 169-183.

Kemps, E., De Rammelaere, S., \& Desmet, T. (2000). The development of 
working memory: Exploring the complementarity of two models. Journal of Experimental Child Psychology, 77, 89-109.

Kieras, D. E., Meyer, D. E., Mueller, S., \& Seymour, T. (1999). Insights into working memory from the perspective of the EPIC architecture for modeling skilled perceptual-motor and cognitive human performance. In A. Miyake \& P. Shah (Eds.), Models of working memory: Mechanisms of active maintenance and executive control (pp. 183-223). Cambridge, England: Cambridge University Press.

Kyllonen, P. C., \& Christal, R. E. (1990). Reasoning ability is (little more than) working-memory capacity? Intelligence, 33, 1-64.

Logan, G. D. (1988). Toward an instance theory of automatization. Psychological Review, 95, 492-527.

Lovett, M. C., Reder, L. M., \& Lebière, C. (1999). Modeling working memory in a unified architecture: An ACT-R perspective. In A. Miyake $\&$ P. Shah (Eds.), Models of working memory: Mechanisms of active maintenance and executive control (pp. 135-182). Cambridge, England: Cambridge University Press.

Miyake, A., Friedman, N. P., Emerson, M. J., Witzki, A. H., Howerter, A., $\&$ Wager, T. D. (2000). The unity and diversity of executive functions and their contributions to complex "frontal lobe" tasks: A latent variable analysis. Cognitive Psychology, 41, 49-100.

Miyake, A., Just, M. A., \& Carpenter, P. A. (1994). Working memory constraints in the resolution of lexical ambiguity: Maintaining multiple interpretations in neutral contexts. Journal of Memory and Language, 33, 175-202.

Monsell, S. (1991). The nature and locus of word frequency effects in reading. In D. Besner \& G. W. Humphreys (Eds.), Basic processes in reading: Visual word recognition (pp. 148-197). Hillsdale, NJ: Erlbaum.

Nairne, J. S. (1988). A framework for interpreting recency effects in immediate serial recall. Memory \& Cognition, 16, 343-352.

Nairne, J. S. (1990). A feature model of immediate memory. Memory \& Cognition, 18, 251-269.

Nairne, J. S. (2002). Remembering over short-term: The case against the standard model. Annual Review of Psychology, 53, 53-81.

Naveh-Benjamin, M., \& Ayres, T. J. (1986). Digit span, reading rate, and linguistic relativity. Quarterly Journal of Experimental Psychology, 38, $739-751$.

Neath, I., \& Nairne, J. S. (1995). Word-length effects in immediate memory: Overwriting trace-decay theory. Psychonomic Bulletin \& Review, 2, 429-441.

Norman, D. A., \& Shallice, T. (1986). Attention to action: Willed and automatic control of behavior. In R. J. Davidson, G. E. Schwartz, \& D. Shapiro (Eds.), Consciousness and self-regulation (Vol. 4, pp. 1-18). New York: Plenum.

Page, M. P. A., \& Norris, D. (1998). The primacy model: A new model of immediate serial recall. Psychological Review, 105, 761-781.

Pascual-Leone, J. A. (1970). A mathematical model for the transition rule in Piaget's developmental stage. Acta Psychologica, 32, 301-345.

Pascual-Leone, J. A. (1978). Compounds, confounds, and models in developmental information processing: A reply to Trabasso and Foellinger. Journal of Experimental Child Psychology, 26, 18-40.

Pashler, H. (1998). The psychology of attention. Cambridge, MA: MIT Press.
Rohrer, D., \& Pashler, H. (2003). Concurrent task effects on memory retrieval. Psychonomic Bulletin \& Review, 10, 96-103.

Rohrer, D., Pashler, H., \& Etchegaray, J. (1998). When two memories can and cannot be retrieved concurrently. Memory \& Cognition, 26, 731739.

Rosen, V. M., \& Engle, R. W. (1997). The role of working memory capacity in retrieval. Journal of Experimental Psychology: General, 126, 211-227.

Rubinstein, J. S., Meyer, D. E., \& Evans, J. E. (2001). Executive control of cognitive processes in task switching. Journal of Experimental Psychology: Human Perception and Performance, 27, 763-797.

Schouten, J. F., Kalsbeek, J. W. H., \& Leopold, F. F. (1960). On the evaluation of perceptual and mental load. Ergonomics, 5, 251-260.

Shah, P., \& Miyake, A. (1996). The separability of working memory resources for spatial thinking and language processing: An individual differences approach. Journal of Experimental Psychology: General, $125,4-27$.

Shah, P., \& Miyake, A. (1999). Models of working memory: An introduction. In A. Miyake \& P. Shah (Eds.), Models of working memory: Mechanisms of active maintenance and executive control (pp. 1-27). Cambridge, England: Cambridge University Press.

Shute, V. J. (1991). Who is likely to acquire programming skills? Journal of Educational Computing Research, 7, 1-24.

Siegler, R. S. (1996). Emerging minds: The process of change in children's thinking. Oxford, England: Oxford University Press.

Siegler, R. S., \& Shrager, J. (1984). Strategy choices in addition and subtraction: How do children know what to do? In C. Sophian (Ed.), Origins of cognitive skills (pp. 229-293). Hillsdale, NJ, Erlbaum.

Smith, M. M., \& Scholey, K. A. (1992). Determining spatial span: The role of movement time and articulation rate. Quarterly Journal of Experimental Psychology, 45(A), 479-501.

Towse, J. N., \& Hitch, G. J. (1995). Is there a relationship between task demand and storage space in tests of working memory capacity? Quarterly Journal of Experimental Psychology, 48(A), 108-124.

Towse, J. N., Hitch, G. J., \& Hutton, U. (1998). A reevaluation of working memory capacity in children. Journal of Memory and Language, 39, 195-217.

Towse, J. N., Hitch, G. J., \& Hutton, U. (2000). On the interpretation of working memory spans in adults. Memory \& Cognition, 28, 341-348.

Towse, J. N., Hitch, G. J., \& Hutton, U. (2002). On the nature of the relationship between processing activity and item retention in children. Journal of Experimental Child Psychology, 82, 156-184.

Towse, J. N., \& Houston-Price, C. M. T. (2001). Reflections on the concept of central executive. In J. Andrade (Ed.), Working memory in perspective (pp. 240-260). Philadelphia: Psychology Press.

Turner, M. L., \& Engle, R. W. (1989). Is working memory task dependent? Journal of Memory and Language, 28, 127-154.

Zbrodoff, N. J., \& Logan, G. D. (1986). On the autonomy of mental processes: A case study of arithmetic. Journal of Experimental Psychology: General, 115, 118-130.

Received September 24, 2002

Revision received October 24, 2003

Accepted October 27, 2003 